QUARTERLY OF APPLIED MATHEMATICS

VOLUME LXVIII, NUMBER 2

JUNE 2010, PAGES 375-393

S 0033-569X(10)01154-7

Article electronically published on February 19, 2010

\title{
TOPOLOGICAL RESOLUTION OF RIEMANN PROBLEMS FOR PAIRS OF CONSERVATION LAWS
}

\author{
$\mathrm{BY}$ \\ ARTHUR V. AZEVEDO (Departamento de Matemática, Universidade de Brasília, 70910-900 \\ Brasília, DF, Brazil), \\ CESAR S. ESCHENAZI (Departamento de Matemática, Universidade Federal de Minas Gerais, \\ Avenida Antônio Carlos 6627, 31270-901 Belo Horizonte, MG, Brazil), \\ DAN MARCHESIN (Instituto de Matemática Pura e Aplicada, Estrada D. Castorina 110, 22460-320 \\ Rio de Janeiro, RJ, Brazil), \\ AND \\ CARlos F. B. PAlmeira (Departamento de Matemática, Pontifícia Universidade Católica do \\ Rio de Janeiro, Rua Marquês de São Vicente 225, 22453-900 Rio de Janeiro, RJ, Brazil)
}

\begin{abstract}
The structure of Riemann solutions to certain systems of conservation laws can be so complicated that the classical constructions are unable to establish their global existence and stability. For systems of two conservation laws, classically the local solution is found by intersecting two wave curves specified by the Riemann data. The intersection point represents the intermediate constant state that typically appears in such solutions. In this paper, we construct the wave curves in a three-dimensional manifold which is globally foliated by shock curves, and where rarefaction and composite curves are naturally defined. The main innovation in this paper is the construction of a differentiable two-dimensional manifold of intermediate states; the local classical construction is replaced by finding the intersection of a wave curve with the intermediate manifold; a transversality argument guarantees the stability of the Riemann solution. The new

Received October 23, 2008.

2000 Mathematics Subject Classification. Primary 35L65, 58J45, 35L60, 57R99.

Key words and phrases. Conservation laws, Riemann problem, wave curves, wave manifold, intermediate surface.

The first author was supported in part by FEMAT under Grant 04/10, UnB under Grant FUNPE 2005, and CNPq/PADCT under Grant 620029/2004-8.

The second author was supported in part by CNPq under Grant 151363/2007-2 and FAPERJ under Grant E-26/150533/2007.

The third author was supported in part by CNPq under Grants 472067/2006-0, 304168/2006-8 and 491148/2005-4, and by FAPERJ under Grants E-26/152.525/2006, E-26/110.310/2007.

E-mail address: arthur@mat.unb.br

E-mail address: cesar@mat.ufmg.br

E-mail address: marchesi@impa.br

E-mail address: fredpalm@mat.puc-rio.br
\end{abstract}


construction has the potential of establishing structural stability properties globally, i.e., for all initial data. This is its main advantage over the classical construction, which is intrinsically local. The construction presented in this work is demonstrated for a family of quadratic polynomial flux functions and their perturbations.

1. Introduction. Lax's well known theorem establishing the existence and stability of Riemann solutions for systems of conservation laws under certain hypotheses is simple and elegant because shocks and rarefaction waves are parametrized by curves that form a local coordinate system. The rarefaction curves are the integral curves of a line field in state space, so they foliate state space and form a coordinate system naturally, which can be global. However, shock curves do not foliate state space, so they can only be part of an approximate coordinate system, which is necessarily local. This is the main obstacle in attempting to generalize Lax's construction so that it becomes global. We have been able to replace state space by a higher-dimensional space that includes directions. In this space, called the wave manifold, both rarefaction curves and shock curves form foliations (possibly with singularities). In this work we show how one can use the wave manifold to generalize both Lax's and Liu's constructions of Riemann solutions for systems of two conservation laws, by introducing the new concept of intermediate surfaces, which replace wave curves. These surfaces are necessary because we found out that wave curves are not nice objects in the wave manifold. We show how Riemann solutions can be constructed by intersecting the intermediate surfaces determined by the left and right Riemann data. We show that this construction is stable under perturbations of data and of flux functions. Because shocks and rarefactions are foliations, there is no obstacle to constructing the Riemann solutions globally, i.e., for all Riemann data. The global construction is the object of future work.

Let us backtrack; the solution of the Riemann problem for a $2 \times 2$ system of conservation laws

with initial data

$$
U_{t}+F(U)_{x}=0
$$

$$
U(x, t=0)= \begin{cases}U_{L} & \text { if } x<0 \\ U_{R} & \text { if } x>0,\end{cases}
$$

where $U=U(x, t)=(u(x, t), v(x, t))^{T}$ and $F(u, v)=(f(u, v), g(u, v))^{T}$ in $C^{3}$, is usually constructed by using appropriately selected shock, rarefaction and composite rarefactionshock curves to build the wave curve from the state $U_{L}$, as well as the reverse wave curve from $U_{R}$. We obtain the parametrization of the Riemann solution as a sequence of curve arcs connecting the left and right states, with speed increasing from left to right; this is an important property that arises from the definition of solutions of (1.1)-(1.2), which are functions of $x / t$.

The solution is constructed by intersecting the wave curve from $U_{L}$ with the reverse wave curve from $U_{R}$. Provided that the speed of the fastest wave from $U_{L}$ is lower than the speed of the slowest wave from $U_{R}$, this intersection determines the constant intermediate state in the Riemann solution. When the geometry of the wave curves is non-trivial, the procedure of finding the intersection is unwieldy to determine the global structure of the solution. 
In order to construct this solution, rather than utilizing the wave curves in the planar state space, we advocate the usage of a 3 -dimensional wave manifold $\mathcal{M}^{3}$, which was introduced for $2 \times 2$ quadratic systems in [10] and generalized to $n \times n$ systems in 8 .

In the current work we show how this geometric framework enables the construction and the study of structural stability of Riemann solutions. Curves that parametrize rarefactions, shocks and rarefaction-shock (or composite) waves for $2 \times 2$ quadratic systems were studied in [15, 10, 44, and 2] in this framework. Neither the entropy admissibility of shock waves nor the geometric consistency of wave sequences were taken into account in those early works. In the current paper we introduce Liu's entropy admissibility criterion within the wave manifold context.

In order to parametrize Riemann solutions in the wave manifold, we construct a 2dimensional surface associated to $U_{L}$, representing intermediate constant states of the Riemann solutions within the wave manifold. This intermediate surface is intersected with the reverse wave curve associated to $U_{R}$, also built in the wave manifold. Since this construction is done in a three-dimensional manifold, transversality is frequent and it implies structural stability of the Riemann solution.

We will assume that the flux functions satisfy certain regularity conditions, which are valid for conservation laws with quadratic flux functions such as the models considered in [15], [10], and [4. We have studied quadratic flux functions because they are the first nontrivial case and because of their importance in applications such as magnetohydrodynamics and elasticity; see [17] and [18.

This paper is organized as follows. In Section 2 we recall the definition of the wave manifold $\mathcal{M}^{3}$ and extend to this manifold the concepts of all the relevant curves: Hugoniot, Hugoniot' ${ }^{\prime}$, rarefaction and composite curves. We also distinguish the characteristic, sonic and sonic' surfaces [8], and discuss their relationships. In Section 3 we extend to $\mathcal{M}^{3}$ Liu's admissibility criterion and introduce local 1-shock and local reverse 2-shock curves. We also show that this admissibility criterion in $\mathcal{M}^{3}$ implies Lax's inequalities for the shocks studied here. In Section 4 we introduce the concept of admissible rarefactions and composite curves and construct the wave curves in $\mathcal{M}^{3}$. In Section 5 we make use of the wave curves to introduce the intermediate surface $\mathcal{W}^{\prime}$. These surfaces and curves are used in Section 6 to construct Riemann solutions in $\mathcal{M}^{3}$. Technical proofs are presented in the appendices.

2. Preliminaries. The solution of the Riemann problem for (1.1)-(1.2) is a sequence of shock waves, rarefaction waves and constant states. In this paper we will consider Riemann solutions with only one intermediate constant state, separating slow waves from fast waves.

Shocks between states $U$ and $U^{\prime}$ are certain discontinuous solutions of (1.1) that satisfy the Rankine-Hugoniot condition

$$
F(U)-F\left(U^{\prime}\right)=s\left(U-U^{\prime}\right),
$$

where $U$ and $U^{\prime}$ in $\mathbb{R}^{2}$ are the states at the left and right ends of the shock and $s=s\left(U, U^{\prime}\right)$ is the propagation speed of the shock. Besides (2.1), shocks satisfy other restrictions that will be discussed later. 
Writing $U=(u, v)^{T}$ and $U^{\prime}=\left(u^{\prime}, v^{\prime}\right)^{T}$ and eliminating $s$ from the two equations in (2.1), one obtains

$$
H\left(u, v, u^{\prime}, v^{\prime}\right)=\left(f(u, v)-f\left(u^{\prime}, v^{\prime}\right)\right)\left(v-v^{\prime}\right)-\left(g(u, v)-g\left(u^{\prime}, v^{\prime}\right)\right)\left(u-u^{\prime}\right)=0 .
$$

This equation defines a set $\mathcal{P}$ in $\left(U, U^{\prime}\right)$-space; its diagonal $U=U^{\prime}$ is denoted by $\Delta$. Provided zero is a regular value for $\left.H\right|_{\mathbb{R}^{4}-\Delta}, \mathcal{P}$ is a 3 -dimensional manifold which is singular along $\Delta$. The fact that $\Delta$ is contained in $\mathcal{P}$ reflects the fact that constant solutions $U=U^{\prime}$ always satisfy (1.1) and (2.1). For a point $\left(U, U^{\prime}\right)$ in $\mathcal{P}$, we call $U$ its first coordinate and $U^{\prime}$ its last coordinate. We can use (2.1) to obtain $s$ as a real function defined in $\mathcal{P}$ outside of $\Delta$. In order to cope with the singular set $\Delta$, we perform a blow up of $\mathcal{P}$ along $\Delta$ to get a 3-dimensional manifold $\mathcal{M}^{3}$, which is called the wave manifold for (1.1). The cornerstone of this process is the usage of the projective angle of the vector $U-U^{\prime}$. We introduce new coordinates $\bar{U}, \bar{V}, X, Y$ and $Z$, defined by equation (A.1). In these coordinates, $\mathcal{M}^{3}$ is given by two equations: $Y=Z X$ and $\mathcal{G}(\bar{U}, \bar{V}, X, Y, Z)=0$, which is equation (2.2) in these coordinates. The diagonal $\Delta$ intersects $\mathcal{M}^{3}$ transversally along a surface called the characteristic, which is denoted by $\mathcal{C}$.

Given a point $\mathcal{U}$ in $\mathcal{M}^{3}$ which corresponds to a point $\left(U, U^{\prime}\right)$ in $\mathcal{P}$, somewhat improperly we will say that $\mathcal{U}=\left(U, U^{\prime}\right)$. This nomenclature is justified by the fact that $\mathcal{M}^{3}-\mathcal{C}$ is diffeomorphic to $\mathcal{P}-\Delta$, and that it simplifies the definitions and statements of properties of Hugoniot and Hugoniot' curves to be introduced now.

Given a point $\mathcal{U}_{-} \in \mathcal{M}^{3}$ with first coordinate $U_{-}=\left(u_{-}, v_{-}\right)$, we define the Hugoniot curve through $\mathcal{U}_{-}$in $\mathcal{M}^{3}$ (denoted by $\operatorname{sh}\left(\mathcal{U}_{-}\right)$) as the set of points $\left(U_{-}, U^{\prime}\right)$, i.e., points with fixed left state $U=U_{-}$. Let $\pi: \mathcal{M}^{3} \longrightarrow \mathbb{R}^{2}$ be defined by $\pi\left(U, U^{\prime}\right)=U^{\prime}$. The projection by $\pi$ of the Hugoniot curve onto the $U^{\prime}$-plane is the classical Rankine-Hugoniot curve (2.2) through $U_{-}$.

For $\mathcal{U}_{+} \in \mathcal{M}^{3}$ with last coordinate $U_{+}$, we also define the Hugoniot' curve (denoted by $\left.s h^{\prime}\left(\mathcal{U}_{+}\right)\right)$as the set of points $\left(U, U_{+}\right)$in $\mathcal{M}^{3}$, i.e., points with fixed right state $U^{\prime}=U_{+}$, as in [8]. It is easy to show that one can define a smooth function $s(\mathcal{U})$ for all $\mathcal{U}$ in $\mathcal{M}^{3}$ which coincides with the classical definition of shock speed, $s=\left(f(u, v)-f\left(u^{\prime}, v^{\prime}\right)\right) /\left(u-u^{\prime}\right)=$ $\left(g(u, v)-g\left(u^{\prime}, v^{\prime}\right)\right) /\left(v-v^{\prime}\right)$, away from $\Delta$.

One can show that Hugoniot curves foliate $\mathcal{M}^{3}$, except at certain points of singularity, which form the so-called secondary bifurcation locus; see [8] and [10]. A similar remark is valid for Hugoniot ${ }^{\prime}$ curves. The Hugoniot and Hugoniot' curves were (somewhat improperly) called shock and shock' curves in [8], [10] and [4].

The characteristic surface $\mathcal{C}$ represents infinitesimally small shocks $U \cong U^{\prime}$; one can show that $s$ extends to $\mathcal{C}$ smoothly and equals a real eigenvalue of $d F(U)$, i.e., a characteristic speed of (1.1), hence the name of this surface. To each point $\mathcal{U} \in \mathcal{C}$ one associates either the slow characteristic speed $\lambda_{s}(U)$ or the fast characteristic speed $\lambda_{f}(U)$, defined as the eigenvalues $\lambda_{s}(U) \leq \lambda_{f}(U)$ of $d F(U)$.

The image of $\mathcal{C}$ by $\pi$ is called the hyperbolic region. The complement of the hyperbolic region is called the elliptic region $\mathcal{E} \mathcal{R}$. The boundary between the elliptic and hyperbolic regions is a locus $E$. The preimage $\mathcal{E}=\pi^{-1}(E)$ is the singular set of the map $\pi$; see 8]. Since the characteristic speeds coincide at both $\mathcal{E}$ and $E$, we call these two loci coincidence loci. The coincidence locus $\mathcal{E}$ splits $\mathcal{C}$ into two submanifolds, $\mathcal{C}_{s}$ and $\mathcal{C}_{f}$, 
associated with $\lambda_{s}$ and $\lambda_{f}$, respectively. For quadratic flux functions the locus $E$ is a conic section. Generically, the coincidence locus $\mathcal{E}$ in $\mathcal{M}^{3}$ consists of fold points of $\pi$.

For $\mathcal{U}_{s} \in \mathcal{C}_{s}$, we have $s\left(\mathcal{U}_{s}\right)=\lambda_{s}\left(U_{s}\right)$; similarly for $\mathcal{U}_{f} \in \mathcal{C}_{f}$, we have $s\left(\mathcal{U}_{f}\right)=\lambda_{f}\left(U_{f}\right)$; see [8]. In this paper, for a given point $\mathcal{U}$ in $\mathcal{M}^{3}$, we define $\mathcal{U}_{s}, \mathcal{U}_{f}, \mathcal{U}_{s}^{\prime}$ and $\mathcal{U}_{f}^{\prime}$ to be the points

$$
\mathcal{U}_{s}=\operatorname{sh}(\mathcal{U}) \cap \mathcal{C}_{s}, \quad \mathcal{U}_{f}=\operatorname{sh}(\mathcal{U}) \cap \mathcal{C}_{f}, \quad \mathcal{U}_{s}^{\prime}=s h^{\prime}(\mathcal{U}) \cap \mathcal{C}_{s} \quad \text { and } \quad \mathcal{U}_{f}^{\prime}=s h^{\prime}(\mathcal{U}) \cap \mathcal{C}_{f} .
$$

Using the classical notation of conservation laws, for $\mathcal{U}=\left(U, U^{\prime}\right)$ the following equalities hold: $s\left(\mathcal{U}_{s}\right)=\lambda_{s}(U), s\left(\mathcal{U}_{f}\right)=\lambda_{f}(U), s\left(\mathcal{U}_{s}^{\prime}\right)=\lambda_{s}\left(U^{\prime}\right)$, and $s\left(\mathcal{U}_{f}^{\prime}\right)=\lambda_{f}\left(U^{\prime}\right)$.

REMARK 2.1. Following the notation introduced above, for a given point $\mathcal{U}$ in $\mathcal{M}^{3}$, if $\mathcal{U}=\mathcal{U}_{s}=\mathcal{U}_{s}^{\prime}$, then $\mathcal{U}_{f}^{\prime}=\mathcal{U}_{f}$. A similar equality is obtained for $\mathcal{U}=\mathcal{U}_{f}=\mathcal{U}_{f}^{\prime}$.

Rarefaction waves are smooth solutions of (1.1) of the form $U(x / t)$. They are parametrized by rarefaction curves, which lie in the characteristic surface $\mathcal{C}$. This surface is foliated by integral curves of a line field, called the rarefaction line field $[15,8$. The projections of this line field belonging to $\mathcal{C}_{s}$ and to $\mathcal{C}_{f}$ are the eigenvectors of $d F$ corresponding to $\lambda_{s}$ and $\lambda_{f}$, respectively. As we will see in Section 4.1, the rarefaction curves in $\mathcal{C}$ are oriented arcs of such integral curves. The projections of rarefaction curves onto the $U^{\prime}$-plane produce classical rarefaction curves.

We introduce the following simplifying assumptions, which are used only in this paper:

Assumption 2.1. We will deal only with points $\mathcal{U}=\left(U, U^{\prime}\right)$ in $\mathcal{M}^{3}$ with $U$ and $U^{\prime}$ in the hyperbolic region, so that the points $\mathcal{U}_{s}$ and $\mathcal{U}_{f}$ in (2.3) always exist.

Assumption 2.2. We consider only Hugoniot and Hugoniot' curves that are diffeomorphic to $\mathbb{R}$; of course, they do not contain points in the secondary bifurcation locus.

The critical points of the speed function $s$ restricted to Hugoniot curves define a surface called sonic, denoted by Son. Similarly, the critical points of $s$ restricted to Hugoniot' curves define a surface called sonic', denoted by $S^{\prime} n^{\prime}[10,8$. The intersection of Son and $S$ Sn $^{\prime}$ consists of a curve in $\mathcal{C}$ called the inflection locus $\mathcal{I}$ and sometimes another curve outside of $\mathcal{C}$ called the double contact locus $\mathcal{D}$ [8]. In the classical context, the inflection locus is the curve where the characteristic speed reaches an extremum along rarefaction curves, while the double sonic locus is the curve where the shock speed is equal to the characteristic speeds at both the left and the right states.

REMark 2.2. By the Bethe-Wendroff theorem (stated in Appendix (D), if $\mathcal{U}=\left(U, U^{\prime}\right)$ lies in $S o n$, then either $s(\mathcal{U})=\lambda_{s}\left(U^{\prime}\right)$ or $s(\mathcal{U})=\lambda_{f}\left(U^{\prime}\right)$; see 8 and 4 . We denote by $S_{\text {Son }}$ the set of points $\mathcal{U} \in S o n$ such that $s(\mathcal{U})=\lambda_{s}\left(U^{\prime}\right)\left(\operatorname{Son}_{f}\right.$ when $\left.s(\mathcal{U})=\lambda_{f}\left(U^{\prime}\right)\right)$. In the same way, if $\mathcal{U}=\left(U, U^{\prime}\right)$ lies in $S o n^{\prime}$, then either $s(\mathcal{U})=\lambda_{s}(U)$ or $s(\mathcal{U})=\lambda_{f}(U)$. We denote by $S_{o n}^{\prime}$ the set of points $\mathcal{U} \in S_{\text {on }}^{\prime}$ such that $s(\mathcal{U})=\lambda_{s}(U)\left(\operatorname{Son}_{f}^{\prime}\right.$ when $\left.s(\mathcal{U})=\lambda_{f}(U)\right)$.

A point $\mathcal{U} \in \operatorname{Son}_{s}$ represents a slow right-characteristic discontinuity in the classical language of conservation laws. Similarly, a point $\mathcal{U} \in S o n_{s}^{\prime}$ represents a slow leftcharacteristic discontinuity. Analogous observations hold for points in $\operatorname{Son}_{f}$ and $\operatorname{Son}_{f}^{\prime}$.

Since $s$ along Hugoniot curves is critical on $\operatorname{Son}_{s}$, generically it has an extremum, except at points that define the hysteresis curve; see Prop. 7.7 and Def. 7.8 in [8. As explained there, for a point $\left(U, U^{\prime}\right)$ of the hysteresis curve, Lax's genuine nonlinearity 
condition fails at $U^{\prime}$; in other words, the point $\left(U^{\prime}, U^{\prime}\right)$ lies on the inflection locus in $\mathcal{C}_{s}$. An analogous observation holds for points in $\operatorname{Son}_{f}$ and $\mathcal{C}_{f}$.

For systems with quadratic polynomial fluxes the topological description of rarefaction curves was studied in [15] and [3], of Hugoniot curves in [10], and of composite curves in [4. For such flux functions, $\mathcal{M}^{3}$ is diffeomorphic to a Möbius band times $\mathbb{R}$. As explained in [15], depending on the values of certain parameters in the flux $F$, the system of conservation laws belongs to one of the four cases in the classification of Schaeffer and Shearer [16.

Actually, 4] and [15] use the classification introduced by Sotomayor and Gutierrez in [7. The correspondence is immediate (cases D1,D2,D3.2 and D3.1 of [15] and [4] correspond to cases $I V, I I I, I I$ and $I$ of [16], respectively).

In cases $I-I I I$, Hugoniot curves in the wave manifold generically have three connected components, all diffeomorphic to $\mathbb{R}$. In case $I V$, Hugoniot curves are connected and generically diffeomorphic to $\mathbb{R}$. In all cases, $\mathcal{C}$, Son and $S o n^{\prime}$ are embedded in $\mathcal{M}^{3}$ and diffeomorphic to cylinders.

3. Admissible shocks in $\mathcal{M}^{3}$. In this section we define shock curves, i.e., arcs of Hugoniot curves in $\mathcal{M}^{3}$, consisting of pairs that satisfy certain restrictions. Our motivation is to generalize Liu's construction of Riemann solutions in [9], which involves finding the intersection between 1-wave curves and reverse 2-wave curves.

In 9] Liu extended Oleinik's celebrated condition to strictly hyperbolic systems of conservation laws [14. He required that shock solutions satisfy the entropy condition

$$
s\left(U_{-}, U_{+}\right)<s\left(U_{-}, U^{\prime}\right)
$$

for every $U^{\prime}$ between $U_{-}$and $U_{+}$on the Hugoniot curve from the left state $U_{-}$, which is a simple curve arc under Assumption 2.2, Transversality of the wave curves was ensured globally by an extra condition that fails for quadratic flux functions. Our construction does not require this extra condition.

Remark 3.1. Along a Hugoniot curve the speed $s$ is critical only at Son; therefore where $\operatorname{sh}\left(\mathcal{U}_{-}\right)$crosses $\mathcal{C}$ or $S$ on $^{\prime}$ away from inflection and double contact loci, $s$ is not stationary. Analogously, along a Hugoniot' curve $s$ is critical only at $S o n^{\prime}$; therefore where $s h^{\prime}\left(\mathcal{U}_{+}\right)$crosses $\mathcal{C}$ or $S o n$ away from inflection and double contact loci, $s$ is not stationary.

We will make the following assumption, which is valid for quadratic flux functions and their perturbations, as shown in Appendix B.

Assumption 3.1. Let $\mathcal{V}$ in $\mathcal{M}^{3}$ be a neighborhood of a point $\mathcal{U}$ in $\mathcal{C}$ away from the inflection and the coincidence loci. Let $\mathcal{V}_{1}$ be one of the two connected components of $\mathcal{V}-\mathcal{C}$. In this paper we assume that if $s$ decreases along the Hugoniot curve through $\mathcal{U}$ into $\mathcal{V}_{1}$, then $s$ increases along the Hugoniot' curve through $\mathcal{U}$ into $\mathcal{V}_{1}$. A similar statement is assumed to hold if $s$ increases along the Hugoniot curve into $\mathcal{V}_{1}$.

Based on condition (3.1) in Liu's construction, we define the local 1-shock curve from $\mathcal{U}_{s}$ in $\mathcal{C}_{s}$ as the closure of the maximal arc of the Hugoniot curve starting at $\mathcal{U}_{s}$ with decreasing $s$. In particular, any point $\mathcal{U}=\left(U, U^{\prime}\right)$ inside the local 1-shock curve satisfies

$$
s(\mathcal{U})<s\left(\mathcal{U}_{s}\right)=\lambda_{s}(U) .
$$




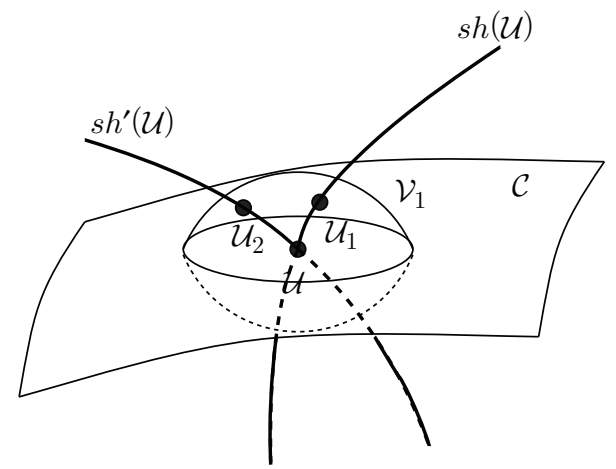

FIG. 1. Illustration of Assumption 3.1 If $s(\mathcal{U})>s\left(\mathcal{U}_{1}\right)$, then $s(\mathcal{U})<s\left(\mathcal{U}_{2}\right)$.

We denote the local 1-shock curve from $\mathcal{U}_{s}$ by $\mathcal{S}_{1}\left(\mathcal{U}_{s}\right)$. We prove now that discontinuities parametrized by local 1-shock curves satisfy Lax's shock inequalities (3.3), under the additional hypothesis that in the local 1-shock curve there are no hysteresis points. This restriction is somewhat weaker than Lax's genuine nonlinearity requirement.

First we prove the inequalities locally. Taking a point $\mathcal{U}$ in $\mathcal{C}_{s}$, from Remark 2.1 we have $\mathcal{U}=\mathcal{U}_{s}=\mathcal{U}_{s}^{\prime}$ and $\mathcal{U}_{f}=\mathcal{U}_{f}^{\prime}$. The shock speed satisfies $s(\mathcal{U})=s\left(\mathcal{U}_{s}\right)=s\left(\mathcal{U}_{s}^{\prime}\right)<$ $s\left(\mathcal{U}_{f}\right)=s\left(\mathcal{U}_{f}^{\prime}\right)$. Considering another point $\mathcal{U}$ in $\mathcal{V}_{1}$ near $\mathcal{U}_{s}$ in the local 1-shock curve through $\mathcal{U}_{s}$, we have $\mathcal{U}_{s}^{\prime}$ near $\mathcal{U}_{s}, \mathcal{U}_{f}^{\prime}$ near $\mathcal{U}_{f}$ and, from (3.2), $s(\mathcal{U})<s\left(\mathcal{U}_{s}\right)$. Assumption 3.1 implies that $s\left(\mathcal{U}_{s}^{\prime}\right)<s(\mathcal{U})$. It follows from the continuity of $s$ that $s(\mathcal{U})<s\left(\mathcal{U}_{f}^{\prime}\right)$.

In summary, in a neighborhood of $\mathcal{C}_{s}$, points in a local 1-shock curve satisfy Lax's criterion for admissible 1-shocks:

$$
s(\mathcal{U})<s\left(\mathcal{U}_{s}\right)=\lambda_{s}(U) \quad \text { and } \quad \lambda_{s}\left(U^{\prime}\right)=s\left(\mathcal{U}_{s}^{\prime}\right)<s(\mathcal{U})<s\left(\mathcal{U}_{f}^{\prime}\right)=\lambda_{f}\left(U^{\prime}\right) .
$$

Actually, any point in a local 1-shock curve satisfies (3.3). This follows from the fact that the first point $\mathcal{U}$ at which the second or third inequality in (3.3) is violated, i.e., where $s(\mathcal{U})=s\left(\mathcal{U}_{s}^{\prime}\right)$ or $s(\mathcal{U})=s\left(\mathcal{U}_{f}^{\prime}\right)$, lies in Son. Because of our additional restriction, at this point $s$ indeed reaches an extremum along the Hugoniot curve, reversing the direction of decreasing $s$, so that the arc ends. If neither the second nor the third inequality in (3.3) is violated, the Hugoniot curve through $\mathcal{U}$ in $\mathcal{V}_{1}$ does not reach the sonic surface, i.e., $s$ decreases along this curve so that the first inequality in (3.3) is preserved.

We remark that if Lax's requirement that the flux function $F$ be genuinely nonlinear is also imposed, then Liu's condition and Lax's criterion are equivalent.

In general, there exist non-local shock curves. Usually, they consist of arcs of points representing shocks that satisfy other admissibility criteria, such as the existence of viscous profiles [6, 12. Often, under appropriate conditions the several criteria are equivalent for local shock curves. Non-local shock curves will not be considered here, except in Theorem 5.1. We have proven the following lemma that describes local 1-shock curve arcs.

Lemma 3.2. Generically, a local 1-shock curve is an arc starting at $C_{s}$ that either $(i)$ stops at $S o n$ or $(i i)$ is unbounded. 
The projection by $\pi$ of the local 1-shock curve with left state $\mathcal{U}_{L}$ (i.e., with first coordinate $U_{L}$ ) onto the state space $U^{\prime}$ consists of classical Lax shocks from $U_{L}$ associated to family 1 , or 1 -shocks. We denote this projection by $S_{1}\left(U_{L}\right)$.

We remark that a local 1-shock curve $\mathcal{S}_{1}\left(\mathcal{U}_{s}\right)$ does not intersect the surface $S o n^{\prime}$. In fact, if $\mathcal{U} \in \mathcal{S}_{1}\left(\mathcal{U}_{s}\right) \cap S o n^{\prime}$, then $s(\mathcal{U})=s\left(\mathcal{U}_{s}\right)$. As $s$ is decreasing along $\mathcal{S}_{1}\left(\mathcal{U}_{s}\right), s$ must reach a minimum before $\mathcal{S}_{1}\left(\mathcal{U}_{s}\right)$ intersects $S o n^{\prime}$. Since critical points of the shock speed $s$ are at $S o n$, by Lemma 3.2 the curve $\mathcal{S}_{1}\left(\mathcal{U}_{s}\right)$ stops at this point.

Based on (3.1) in Liu's construction, we define the local reverse 2-shock curve from $\mathcal{U}_{f}$ in $\mathcal{C}_{f}$ as the closure of the maximal arc of the Hugoniot curve starting at $\mathcal{U}_{f}$ with increasing $s$. In particular, any point $\mathcal{U}$ in a local reverse 2 -shock curve satisfies

$$
s(\mathcal{U})>s\left(\mathcal{U}_{f}\right) .
$$

We denote a local reverse 2-shock curve by $\mathcal{S}_{2}^{r}\left(\mathcal{U}_{f}\right)$. Again, in regions of strict hyperbolicity our construction of local reverse 2 -shock curves implies that Lax's inequalities for family 2 shocks hold:

$$
\lambda_{s}\left(U^{\prime}\right)=s\left(\mathcal{U}_{s}^{\prime}\right)<s(\mathcal{U})<s\left(\mathcal{U}_{f}^{\prime}\right)=\lambda_{f}\left(U^{\prime}\right) \quad \text { and } \quad \lambda_{f}(U)=s\left(\mathcal{U}_{f}\right)<s(\mathcal{U}) .
$$

Given a point $\mathcal{U}_{*} \in \mathcal{C}_{f}$, we emphasize that $s h^{\prime}\left(\mathcal{U}_{*}\right)$ and $\mathcal{S}_{2}^{r}\left(\mathcal{U}_{*}\right)$ do not coincide. While the former is obtained by fixing the right coordinate of $\mathcal{U}_{*}$, the latter is obtained by fixing the left coordinate of $\mathcal{U}_{*}$; of course these two curves are not tangent at $\mathcal{U}_{*}$.

Generically, a local reverse 2 -shock curve is an arc starting at $\mathcal{C}_{f}$ that either stops at Son or is unbounded. The proof of this fact is similar to the proof of Lemma 3.2

We remark that in the classical context of conservation laws, a point $\left(U_{R}, U_{M}\right)$ in $\mathcal{S}_{2}^{r}\left(\mathcal{U}_{R}\right)$ represents a Lax 2-shock from $U_{M}$ to $U_{R}$.

4. Wave curves. In general, wave curves in $\mathcal{M}^{3}$ consist of arcs of admissible shock curves, rarefaction curves and composite curves of the same family. In this work, we will focus on local wave curves, which consist of local shock, rarefaction and composite arcs. The admissible local shock curves in $\mathcal{M}^{3}$ were described in Section 3 We describe the construction of admissible local rarefaction curves and admissible local composite curves in $\mathcal{M}^{3}$ in Subsection 4.1. We construct the local wave curves used in this paper in Subsection 4.2. The projections by $\pi$ of these local wave curves onto $\left(u^{\prime}, v^{\prime}\right)$-space are some of the wave curves described by Liu in [9]; here we consider wave curves containing only local shock curves and local rarefaction and composite curves (defined below) to shorten the list of cases. Wave curves in the classical context were studied in [9] and [11].

4.1. Admissible rarefaction curves and composite curves. In this section we describe the rarefaction curves and composite curves; in the case of quadratic flux functions these curves were described in [15] and [4, respectively.

The local 1-rarefaction curve from $\mathcal{U}_{s} \in \mathcal{C}_{s}$ is the arc of the rarefaction curve starting at $\mathcal{U}_{s}$, with $s$ increasing away from $\mathcal{U}_{s}$. We denote this curve by $\mathcal{R}_{1}\left(\mathcal{U}_{s}\right)$. The speed along $\mathcal{R}_{1}\left(\mathcal{U}_{s}\right)$ is the 1-characteristic speed. This curve starts at $\mathcal{U}_{s}$ and either is unbounded or stops at the 1-inflection or coincidence fold loci. The latter case will not occur in the examples presented in this paper. 
At the inflection locus the 1-characteristic speed along the 1-rarefaction curve is critical; generically it attains a maximum. Of course the projection by $\pi$ of a 1-rarefaction curve onto state space is a classical 1-rarefaction curve.

The local reverse 2-rarefaction curve from $\mathcal{U}_{f} \in \mathcal{C}_{f}$ is the arc of the rarefaction curve starting at $\mathcal{U}_{f}$, with $s$ decreasing away from $\mathcal{U}_{f}$. We denote this arc of rarefaction by $\mathcal{R}_{2}^{r}\left(\mathcal{U}_{f}\right)$. The speed along $\mathcal{R}_{2}^{r}\left(\mathcal{U}_{f}\right)$ is the 2 -characteristic speed. As for the 1-rarefaction curve, the local reverse 2-rarefaction curve starts at $\mathcal{U}_{f}$ and either is unbounded or stops (at the 2-inflection or fold loci). At the inflection locus the fast eigenvalue is critical along $\mathcal{R}_{2}^{r}\left(\mathcal{U}_{f}\right)$.

Composite curves parametrize successions of a rarefaction wave and a shock wave with no intermediate region of constant state. The reader should recall the definition and properties of $S$ on $^{\prime}$ in Remark 2.2. To construct the composite curves in $\mathcal{M}^{3}$, we use the map $\psi: \operatorname{Son}^{\prime} \longrightarrow \mathcal{C}$, introduced in [4, such that for each point $\mathcal{U} \in \operatorname{Son}^{\prime}, \psi(\mathcal{U})$ is the unique point in $s h(\mathcal{U}) \cap \mathcal{C}$ satisfying $s(\psi(\mathcal{U}))=s(\mathcal{U})$. In the same way, we define the map $\psi^{\prime}:$ Son $\longrightarrow \mathcal{C}$.

The 1-composite curve from $\mathcal{U}_{s}$ is the set of points $\mathcal{U}$ in $S o n^{\prime}$ such that $\psi(\mathcal{U}) \in \mathcal{R}_{1}\left(\mathcal{U}_{s}\right)$. In other words, 1 -composites are the pullbacks by $\psi$ of 1-rarefaction curves. The local 1-composite curve is the arc of the 1-composite starting at the 1-inflection locus with decreasing $s$. We denote this curve by $\mathcal{C O}_{1}\left(\mathcal{U}_{s}\right)$.

The reverse 2-composite curve from $\mathcal{U}_{f}$ is the set of points $\mathcal{U} \in S \operatorname{Son}^{\prime}$ such that $\psi(\mathcal{U}) \in$ $\mathcal{R}_{2}^{r}\left(\mathcal{U}_{f}\right)$. In other words, the reverse 2-composite curves are the pullbacks by $\psi$ of reverse 2-rarefaction curves. The local reverse 2-composite curve from $\mathcal{U}_{f}$ is the arc of the reverse 2 -composite starting at the 2 -inflection locus with increasing $s$. We denote this curve by $\mathcal{C O}_{2}^{r}\left(\mathcal{U}_{f}\right)$.

4.2. Local wave curves. In this paper, we define the local 1-wave curve in $\mathcal{M}^{3}$ from a point $\mathcal{U}_{L}$ in $\mathcal{C}_{s}$, denoted by $\mathcal{W}_{1}\left(\mathcal{U}_{L}\right)$, as a succession of arcs of the following kinds: local 1-rarefaction, local 1-shock and local 1-composite arcs, all from $U_{L}$. We list now the transitions between local arcs. These transitions are only continuous in $\mathcal{M}^{3}$.

(a) 1-rarefaction to 1-shock. In this case we consider a 1-rarefaction arc and a 1-shock arc both starting at the same point $\mathcal{U}_{s}$ in $\mathcal{C}_{s}$; the speed $s$ increases along the rarefaction arc and decreases along the shock arc.

(b) 1-rarefaction to 1-composite. When the 1-rarefaction arc reaches the inflection locus, we continue with a local 1-composite arc (remember that composite curves are defined on the $S o n^{\prime}$ surface, and that the inflection locus is contained in this surface).

There are also transitions involving non-local wave arcs; we list those to which Theorem 5.1 applies. We warn the reader that there are a few other transitions in general, which will not be treated here.

(c) 1-composite to (non-local) 1-shock. When the 1-composite reaches the point $\mathcal{P}_{1}$ in Son' such that $s\left(\mathcal{P}_{1}\right)=s\left(\mathcal{U}_{L}\right)$, the 1-wave curve continues with a (non-local) 1 -shock curve starting at $\mathcal{P}_{1}$.

(d) 1-shock to (non-local) 1-rarefaction. Actually, in this case, we use an arc of the Hugoniot' curve as an auxiliary element. When the 1-shock arc reaches $S o n$ at a point $\mathcal{Q}_{1}$, we construct the auxiliary Hugoniot' arc from $\mathcal{Q}_{1}$, which intersects the 
characteristic surface at a point $\mathcal{Q}_{2}$ such that $s\left(\mathcal{Q}_{1}\right)=s\left(\mathcal{Q}_{2}\right)$. From $\mathcal{Q}_{2}$ the 1-wave curve continues with a (non-local) 1-rarefaction arc.

(e) 1-composite to (non-local) 1-rarefaction. In this case we also use an arc of the Hugoniot' curve as an auxiliary element. When the 1-composite arc reaches the double contact locus at a point $\mathcal{Q}_{1}$, we construct the auxiliary Hugoniot' ${ }^{\prime}$ arc, which intersects the characteristic surface at a point $\mathcal{Q}_{2}$ such that $s\left(\mathcal{Q}_{2}\right)=s\left(\mathcal{Q}_{1}\right)$. From this point the 1-wave continues with a (non-local) 1-rarefaction arc.

Notice that the transitions in cases (d) and (e) are not even continuous in $\mathcal{M}^{3}$.

As we are considering transitions from arcs of family 1 to arcs of family 1 , it does not make sense to consider transitions from 1-rarefaction to 1-rarefaction, from 1-shock to 1-shock or from 1-shock to 1-composite.

REMARK 4.1. Projection of the local 1-wave curves, $\mathcal{W}_{1}\left(\mathcal{U}_{L}\right)$, onto the $\left(u^{\prime}, v^{\prime}\right)$-space by $\pi$ gives rise to the 1-wave curves described by Liu in 9. (Actually Liu's wave curves were more general, as they included non-local arcs.)

Similarly we can define the local reverse 2-wave curve from a point $\mathcal{U}_{R}$ in $\mathcal{C}_{f}$, denoted by $\mathcal{W}_{2}^{r}\left(\mathcal{U}_{R}\right)$, as a succession of arcs of the following kinds: local reverse 2-rarefaction, local reverse 2 -shock and local reverse 2-composite arcs, all from $\mathcal{U}_{R}$.

We remark that wave curves in $\mathcal{M}^{3}$ are discontinuous at many transitions from one kind of non-local arc to another, even though their classical projections onto state space are continuous. Thus wave curves in $\mathcal{M}^{3}$ are not good objects. The good generalizations of wave curves from state space to $\mathcal{M}^{3}$ are the intermediate surfaces.

5. The intermediate surface. The Riemann data (1.2) for (1.1) is represented by a general point $\left(U_{L}, U_{R}\right)$ in $\mathbb{R}^{2} \times \mathbb{R}^{2}$. We utilize the wave manifold to find the solution of the Riemann problem $\left(U_{L}, U_{R}\right)$, by finding the intersection of $\mathcal{W}_{2}^{r}\left(\mathcal{U}_{R}\right)$ and a surface denoted by $\mathcal{W}_{1}^{\prime}=\mathcal{W}_{1}^{\prime}\left(\mathcal{U}_{L}\right)$, constructed as follows.

For each point $\mathcal{U}$ in a 1 -shock $\mathcal{S}_{1}\left(\mathcal{U}_{L}\right)$ of the wave curve we consider the Hugoniot' curve through $\mathcal{U}$. As $\mathcal{U}$ moves along the 1-shock arc, the Hugoniot' curves generate a surface denoted by $\mathcal{W}_{\mathcal{S}_{1}}^{\prime}$. In the same way, as $\mathcal{U}$ moves along a 1-rarefaction arc (or 1-composite arc), its Hugoniot' curve generates a surface denoted by $\mathcal{W}_{\mathcal{R}_{1}}^{\prime}$ (or $\mathcal{W}_{\mathcal{C} \mathcal{O}_{1}}^{\prime}$ ). The intermediate surface, denoted by $\mathcal{W}_{1}^{\prime}$, is the union of all pieces of type $\mathcal{W}_{\mathcal{S}_{1}}^{\prime}, \mathcal{W}_{\mathcal{R}_{1}}^{\prime}$, or $\mathcal{W}_{\mathcal{C O}_{1}}^{\prime}$, corresponding to all arcs of the 1 -wave curve.

In order to prove that each piece of $\mathcal{W}_{1}^{\prime}$ is a regular surface, we assume that the coordinates of the flux functions satisfy a regularity condition that is stated precisely in the proof of the following result, which is technical and thus presented in Appendix C.

ThEOREM 5.1. An intermediate surface $\mathcal{W}_{1}^{\prime}$ containing transitions of types (a)-(e) is a 2-dimensional submanifold of $\mathcal{M}^{3}$, i.e., not only is each piece a differentiable surface, but all pieces join differentiably too.

The fact that the pieces join differentiably can also be obtained as a consequence of the Bethe-Wendroff theorem as stated in Furtado's doctoral thesis [5. For completeness we present this version of the theorem in Appendix D. 
Analogously we define the reverse intermediate surface $\mathcal{W}_{2}^{\prime r}=\mathcal{W}_{2}^{\prime r}\left(\mathcal{U}_{R}\right)$, i.e., from

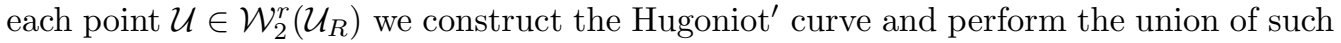
curves. A result analogous to Theorem 5.1 also holds for reverse intermediate surfaces.

We will take advantage of the differentiability of the intermediate surface at transitions of types (a) and (b) in Section 6. The differentiability at transitions of types (c), (d) and (e) will be used in future work, when the locality of shock, rarefaction and composite curves ceases to be required.

6. The construction of the Riemann solution in $\mathcal{M}^{3}$. In order to illustrate the usefulness of intermediate surfaces, we describe in this section the construction of the solution of the Riemann problem with data $\left(U_{L}, U_{R}\right)$. For simplicity, we assume that only local wave curves are involved. We define $\mathcal{U}_{L}$ as the point in $\mathcal{C}_{s}$ with both coordinates equal to $U_{L}$; similarly $\mathcal{U}_{R}$ is the point in $\mathcal{C}_{f}$ with both coordinates equal to $U_{R}$. We will see that the Riemann solution in $\mathcal{M}^{3}$ is represented by a sequence of points (shocks) and curves (rarefactions) with associated speeds that increase from $\mathcal{U}_{L}$ to $\mathcal{U}_{R}$.

From the point $\mathcal{U}_{L}$ we construct the intermediate surface $\mathcal{W}_{1}^{\prime}\left(\mathcal{U}_{L}\right)$ and from the point $\mathcal{U}_{R}$ we construct the reverse 2-wave curve $\mathcal{W}_{2}^{r}\left(\mathcal{U}_{R}\right)$. We assume that the intersection $\mathcal{P}_{2}$ of $\mathcal{W}_{1}^{\prime}\left(\mathcal{U}_{L}\right)$ and $\mathcal{W}_{2}^{r}\left(\mathcal{U}_{R}\right)$ exists and is transversal. Then there is at least one point $\mathcal{P}_{1}$ in $\mathcal{W}_{1}\left(\mathcal{U}_{L}\right) \cap s h^{\prime}\left(\mathcal{P}_{2}\right)$. Notice that $\mathcal{P}_{1}$ and $\mathcal{P}_{2}$ have the same last coordinate, which we call $U_{M}$. In the context of classical Riemann solutions for systems of two equations, $U_{M}$ is the intermediate (or middle) constant state. As mentioned in the introduction, the only case that makes sense is the one where the speed of the fastest wave in $\mathcal{W}_{1}\left(\mathcal{U}_{L}\right)$ is lower than the speed of the slowest wave in $\mathcal{W}_{2}^{r}\left(\mathcal{U}_{R}\right)$, i.e, $s\left(\mathcal{P}_{1}\right)<s\left(\mathcal{P}_{2}\right)$. We will take this inequality as an assumption from now on. Moreover we may regard the part of the Hugoniot ${ }^{\prime}$ curve between $\mathcal{P}_{1}$ and $\mathcal{P}_{2}$ as representing all points in the constant state $U_{M}$ parametrized by their speeds ranging from $s\left(\mathcal{P}_{1}\right)$ to $s\left(\mathcal{P}_{2}\right)$. This stems from the fact that Hugoniot' ${ }^{\prime}$ curves are diffeomorphic to $\mathbb{R}$ (Assumption 2.2).

An equivalent and useful way of obtaining the Riemann solution in $\mathcal{M}^{3}$ is to construct the reverse intermediate surface $\mathcal{W}_{2}^{\prime r}\left(\mathcal{U}_{R}\right)$ and find the intersection $\mathcal{W}_{1}^{\prime}\left(\mathcal{U}_{L}\right) \cap \mathcal{W}_{2}^{\prime r}\left(\mathcal{U}_{R}\right)$. This intersection is a Hugoniot' curve, consisting of points with the same last coordinate $U_{M}$. This curve intersects $\mathcal{W}_{1}\left(\mathcal{U}_{L}\right)$ at the previous point $\mathcal{P}_{1}=\left(U_{L}, U_{M}\right)$. We assume that this intersection occurs transversally. This transversality implies that the point $U_{M}$ depends differentiably on $U_{L}$ and on $U_{R}$. This other way of obtaining Riemann solutions justifies why the intersection between the discontinuous reverse 2-wave curves $\mathcal{W}_{2}^{r}\left(\mathcal{U}_{R}\right)$ and the differentiable intermediate surface $\mathcal{W}_{1}^{\prime}\left(\mathcal{U}_{L}\right)$ gives rise to well posed Riemann solutions: any discontinuity occurs along Hugoniot' curves, so it disappears because its projection onto $U^{\prime}$-space reduces to a point.

Next we describe the nine possible ways in which intersections occur, using the mechanisms introduced above.

Shock-shock intersection. A simple construction occurs when $\mathcal{P}_{1}=\left(U_{L}, U_{M}\right) \in \mathcal{S}_{1}\left(\mathcal{U}_{L}\right)$ and $\mathcal{P}_{2}=\left(U_{R}, U_{M}\right) \in \mathcal{S}_{2}^{r}\left(\mathcal{U}_{R}\right)$. In this case $\mathcal{P}_{1}, \mathcal{P}_{2} \in \mathcal{W}_{\mathcal{S}_{1}}^{\prime}\left(\mathcal{U}_{L}\right)$ and the Riemann solution in $\mathcal{M}^{3}$ is constructed through the Hugoniot' ${ }^{\prime}$ curve connecting $\mathcal{P}_{1}$ with $\mathcal{P}_{2}$. The point $\mathcal{P}_{1}$ represents a 1 -shock from the state $\mathcal{U}_{L}$ to a state $\mathcal{U}_{M}$, the Hugoniot' curve from $\mathcal{P}_{1}$ to $\mathcal{P}_{2}$ represents the constant state $U_{M}$, and the point $\mathcal{P}_{2}$ represents a 2 -shock from the 


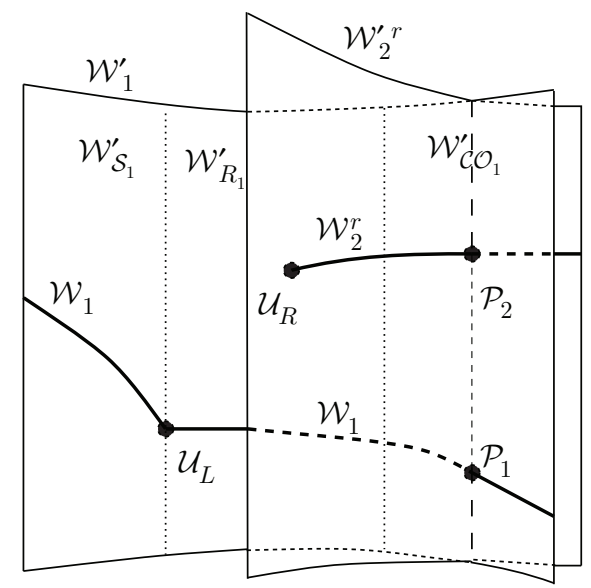

FIG. 2. Riemann solution with $P_{1} \in \mathcal{C O}_{1}\left(\mathcal{U}_{L}\right)$. We illustrate cases where $\mathcal{P}_{2}$ belongs to a shock or to a composite segment of $\mathcal{W}_{2}^{r}\left(\mathcal{U}_{R}\right)$.

state $U_{M}$ to the state $U_{R}$. In the classical context, the Riemann solution is formed by taking a Lax 1-shock from $U_{L}$ to $U_{M}$, followed by a constant state $U_{M}$, followed by a Lax 2-shock from $U_{M}$ to $U_{R}$.

The descriptions of shock-rarefaction, rarefaction-shock and rarefaction-rarefaction intersections are similar to the shock-shock intersection case.

Composite-shock intersection. If $\mathcal{P}_{1}=\left(U_{N}, U_{M}\right) \in \mathcal{C O}_{1}\left(\mathcal{U}_{L}\right)$ and $\mathcal{P}_{2}=\left(U_{R}, U_{M}\right) \in$ $\mathcal{S}_{2}^{r}\left(\mathcal{U}_{R}\right)$, then $\mathcal{P}_{1}, \mathcal{P}_{2} \in \mathcal{W}_{\mathcal{C} \mathcal{O}_{1}}^{\prime}\left(\mathcal{U}_{L}\right)$ and $\mathcal{U}_{R}$ lies on the 2-shock curve from $\mathcal{P}_{2}$. In this case the Riemann solution in $\mathcal{M}^{3}$ is constructed by taking a 1-rarefaction from $\mathcal{U}_{L}$ to a state $\mathcal{U}_{N}=\left(U_{N}, U_{N}\right)$ in $C_{s}$, followed by a segment of 1-shock curve from $\mathcal{U}_{N}$ to the compositeshock intersection $\mathcal{P}_{1} \in S o n^{\prime}$, followed by a Hugoniot' curve from $\mathcal{P}_{1}$ to $\mathcal{P}_{2}=\left(U_{R}, U_{M}\right)$, followed by a 2 -shock from $\mathcal{P}_{2}$ to $\mathcal{U}_{R}$. In the classical context, this solution consists of a 1-rarefaction from the state $U_{L}$ to a state $U_{N}$, followed by a left-characteristic 1-shock from $U_{N}$ to $U_{M}$, followed by a constant state $U_{M}$, followed by a Lax 2-shock from $U_{M}$ to the state $U_{R}$. Figure 2 illustrates this case when the relevant part of $\mathcal{W}_{2}^{r}\left(\mathcal{U}_{R}\right)$ is $\mathcal{S}_{2}^{r}\left(\mathcal{U}_{R}\right)$.

The construction of a composite-rarefaction intersection is similar to the compositeshock case.

Composite-composite intersection. Figure 2 also illustrates this case, when the relevant part of $\mathcal{W}_{2}^{r}\left(\mathcal{U}_{R}\right)$ is $\mathcal{C O}_{2}^{r}\left(\mathcal{U}_{R}\right)$. If $\mathcal{P}_{1}=\left(U_{N}, U_{M}\right) \in \mathcal{C O}_{1}\left(\mathcal{U}_{L}\right)$ and $\mathcal{P}_{2} \in \mathcal{C O}_{2}^{r}\left(\mathcal{U}_{R}\right)$, then $\mathcal{P}_{1}, \mathcal{P}_{2} \in \mathcal{W}_{\mathcal{C O}_{1}}^{\prime}\left(\mathcal{U}_{L}\right)$ and $\mathcal{P}_{2}=\left(U_{O}, U_{M}\right)$. In this case the Riemann solution in $\mathcal{M}^{3}$ is constructed by taking a 1-rarefaction from $\mathcal{U}_{L}$ to $\mathcal{U}_{N}=\left(U_{N}, U_{N}\right)$ in $C_{s}$, followed by a shock curve from $\mathcal{U}_{N}$ to the left-characteristic 1 -shock $\mathcal{P}_{1} \in S o n^{\prime}$, followed by a Hugoniot' curve from $\mathcal{P}_{1}$ to $\mathcal{P}_{2} \in S o n^{\prime}$, followed by a shock curve from $\mathcal{P}_{2}$ to $\mathcal{U}_{O}=\left(U_{O}, U_{O}\right)$ in $\mathcal{C}_{f}$ and then followed by a final 2-rarefaction from $\mathcal{U}_{O}$ to $\mathcal{U}_{R}$. In the classical context, this solution consists of a 1-rarefaction from the state $U_{L}$ to a state $U_{N}$, followed by a left-characteristic 1-shock from $U_{N}$ to $U_{M}$, followed by a constant state $U_{M}$, followed by a right-characteristic 2-shock from $U_{M}$ to $U_{O}$, followed by a 2-rarefaction from $U_{O}$ to the state $U_{R}$. 
The shock-composite and rarefaction-composite intersections can easily be obtained through variations of the constructions presented above.

\section{Appendices.}

A. The wave manifold. In this appendix we introduce the coordinates

$$
\bar{U}=\left(u+u^{\prime}\right) / 2, \quad \bar{V}=\left(v+v^{\prime}\right) / 2, \quad X=u-u^{\prime}, \quad Y=v-v^{\prime}, \quad Z=Y / X .
$$

Substituting (A.1) in (2.2) and eliminating $s$ between the two equations, we perform a blow up process, and the wave manifold $\mathcal{M}^{3}$ is given by $\mathcal{G}(\bar{U}, \bar{V}, X, Y, Z)=0$ (where $\mathcal{G}$ is a map from $\mathbb{R}^{4} \times S^{1}$ to $\mathbb{R}$ ) together with $Y=Z X$. Replacing $Y$ by $Z X$ in the equation $\mathcal{G}=0$, the wave manifold is rewritten as $G(\bar{U}, \bar{V}, X, Z)=0$, where $G$ is a map from $\mathbb{R}^{3} \times S^{1}$ to $\mathbb{R}$. Notice that when we write $Z=Y / X$, we are implicitly assuming that $X \neq 0$. If $X=0$, instead of $Z$ we use $z=1 / Z$ and $X=z Y$. We warn the reader that $\bar{U}$ and $\bar{V}$ are the $U$ and $V$ coordinates utilized in [10] and [4]; these references contain the equations for the characteristic, sonic and sonic' surfaces, as well as the Hugoniot and Hugoniot' curves that follow. The characteristic surface $\mathcal{C}$ is given by

$$
G=0, \quad X=0 .
$$

In the coordinates (A.1), Hugoniot curves are given by

$$
G=0, \quad d G=0, \quad d X+2 d \bar{U}=0, \quad Z d X+X d Z+2 d \bar{V}=0,
$$

and Hugoniot ${ }^{\prime}$ curves are obtained by changing $X$ to $-X$ :

$$
G=0, \quad d G=0, \quad-d X+2 d \bar{U}=0, \quad-Z d X-X d Z+2 d \bar{V}=0 .
$$

Recall that in Section 2 we defined a speed function $s$ in $\mathcal{M}^{3}$. The sonic' surface is the set of points in $\mathcal{M}^{3}$ such that $d G, d s$, and the second and third differential forms in (A.4) are linearly dependent. This condition leads to a zero determinant, so the sonic' surface is given by an equation $S^{\prime}(\bar{U}, \bar{V}, X, Z)=0$, as well as $G=0$. Similarly, the sonic surface is given by an equation $S(\bar{U}, \bar{V}, X, Z)=0$, obtained from $S^{\prime}$ upon replacing $X$ by $-X$.

Parametric equations for Hugoniot curves are obtained by solving in $\bar{U}, \bar{V}$ and $X$ the system

$$
\left\{\begin{array}{c}
G(\bar{U}, \bar{V}, X, Z)=0 \\
\bar{U}+X / 2=u_{s} \\
\bar{V}+Z X / 2=v_{s} .
\end{array}\right.
$$

Remark A.1. Parametric equations for the Hugoniot' curves are found by solving the system obtained from (A.5) upon replacing $X$ by $-X$ and $\left(u_{s}, v_{s}\right)$ by $\left(u_{s}^{\prime}, v_{s}^{\prime}\right)$ in the last two equations.

Following [15], the differential system for rarefactions is given by

$$
\left\{\begin{array}{c}
G(\bar{U}, \bar{V}, X, Z)=0 \\
d G(\bar{U}, \bar{V}, X, Z)=0 \\
Z d \bar{U}-d \bar{V}=0 \\
d X=0 .
\end{array}\right.
$$


Following [4], the differential system for composite curves is given by

$$
\left\{\begin{array}{c}
G(\bar{U}, \bar{V}, X, Z)=0 \\
d G(\bar{U}, \bar{V}, X, Z)=0 \\
\psi^{*}(Z d \bar{U}-d \bar{V})=0 \\
d S^{\prime}(\bar{U}, \bar{V}, X, Z)=0
\end{array}\right.
$$

where $S^{\prime}(\bar{U}, \bar{V}, X, Z)=0$ is the equation of sonic ${ }^{\prime}$ and $\psi$ was defined in Subsection 4.1 when introducing the composite curves.

As an example, the normal form for quadratic flux functions $F$ introduced in [15] is

$$
F(u, v)=\left(v^{2} / 2+b_{1} u^{2} / 2+a_{1} u+a_{2} v, u v-b_{2} v^{2} / 2+a_{3} u+a_{4} v\right)^{T} .
$$

Following [10] and 4], we get $G(\bar{U}, \bar{V}, X, Z)$ as

$$
G(\bar{U}, \bar{V}, X, Z)=\left(1-b_{2} Z-Z^{2}\right) \bar{V}-b_{1} Z \bar{U}-a_{2} Z^{2}-\left(a_{1}-a_{4}\right) Z+a_{3} .
$$

Notice that (A.9) does not depend explicitly on $X$. This fact will play a crucial role in Appendix B] We also notice that for quadratic flux functions, A.5 is a linear system in $\bar{U}, \bar{V}$ and $X$. For the flux function (A.8), the sonic' surface is given by $G=0$ together with

$$
S^{\prime}(\bar{U}, \bar{V}, X, Z)=A_{U}\left(b_{1} \bar{U}+b_{2} \bar{V}+a_{1}-a_{4}\right)+A_{V}\left(V+a_{2}\right)-A_{X} X=0,
$$

where

$A_{U}=2\left(Z^{2}+b 1+1\right), \quad A_{V}=2 Z\left(Z^{2}-b_{2} Z+b_{1}+3\right), \quad A_{X}=-Z^{2}-b_{2}\left(b_{1}+1\right) Z+b_{1}+1$ and $a_{1}, a_{2}, a_{4}, b_{1}$ and $b_{2}$ are the parameters in (A.8). As in [4], the speed $s$ is given by

$$
s=s(\bar{U}, \bar{V}, Z)=Z\left(\bar{V}+a_{2}\right)+\left(b_{1}+1\right) \bar{U}+a_{1} .
$$

B. Verification of Assumption 3.1 for quadratic flux functions. We follow the notation introduced in Appendix $\mathrm{A}$ In $\bar{U}, \bar{V}, X, Z$ coordinates the wave manifold is given by $G=0$, Hugoniot curves are given by (A.3) and Hugoniot' curves are given by (A.4). From equation (A.2) we can represent a point $\mathcal{U} \in \mathcal{C}$ by $\left(\bar{U}_{0}, \bar{V}_{0}, 0, Z_{0}\right)$. Solving system (A.5), the parametric equation for the Hugoniot curve through $\mathcal{U}$ is

$$
\operatorname{sh}(Z)=(\bar{U}=\alpha(Z), \bar{V}=\beta(Z), X=\gamma(Z), Z) .
$$

Using Remark A.1, the parametric equation for the Hugoniot' curve through $\mathcal{U}$ is

$$
s h^{\prime}(Z)=(\bar{U}=\alpha(Z), \bar{V}=\beta(Z), X=-\gamma(Z), Z) .
$$

Here by an abuse of notation we used $s h$ and $s h^{\prime}$ to denote parametric equations for the Hugoniot and Hugoniot' curves.

Let us denote by $\mathcal{V}_{2}$ the other connected component of $\mathcal{V}-\mathcal{C}$. Notice that the $X$ component of $\operatorname{sh}(Z)$ and $s h^{\prime}(Z)$ vanishes at $\mathcal{C}$, i.e., $\gamma\left(Z_{0}\right)=0$, and therefore a point lies either in $\mathcal{V}_{1}$ or in $\mathcal{V}_{2}$ according to the sign of the $X$ component of the parametric equation of the Hugoniot curve through this point; so for any given $Z, \operatorname{sh}(Z)$ and $\operatorname{sh}^{\prime}(Z)$ are in opposite neighborhoods $\mathcal{V}_{i}$. Let us reparametrize $\operatorname{sh}(Z)$ and $s h^{\prime}(Z)$ by a parameter $\xi$ such that $\gamma(\xi=0)=0$, so that the curves for $\xi>0$ are in opposite neighborhoods of 
$\mathcal{V}-\mathcal{C}$. This is done by taking $Z=Z_{0}+\xi$ for $s h$ and $Z=Z_{0}-\xi$ for $s h^{\prime}$. Now we calculate $d s / d \xi$ along $s h$ and $s h^{\prime}$ and check that opposite signs are obtained. As seen in (A.11), the expression of $s$ in $\bar{U}, \bar{V}, X, Z$ coordinates does not depend on $X$, so

$$
\frac{d s}{d \xi}=\left(\frac{\partial s}{\partial \bar{U}} \frac{d \alpha}{d Z}+\frac{\partial s}{\partial \bar{V}} \frac{d \beta}{d Z}+\frac{\partial s}{\partial Z}\right) \frac{\partial Z}{\partial \xi}
$$

for both Hugoniot and Hugoniot' ${ }^{\prime}$ curves. The coefficient of $\partial Z / \partial \xi$ is nonzero because $\mathcal{U}$ is outside of the inflection locus. It follows that the derivative of the speed $s$ has opposite signs along Hugoniot and Hugoniot' ${ }^{\prime}$ curves in the each neighborhood $\mathcal{V}_{i}$.

Although the result is stated for quadratic flux functions $f$ and $g$, by transversality it remains true under $C^{3}$-perturbations of the fluxes in the Whitney topology.

C. Proof of Theorem [5.1, We recall that we are considering only points satisfying Assumptions 2.1 and 2.2. We will use the following two results in the proof of Theorem 5.1. We will omit the proof of the first lemma because it is trivial.

Lemma C.1. Given a 3-dimensional manifold with non-singular vector field $V$, let $\Phi$ be its Poincaré map between two transversal sections $\Sigma_{1}$ and $\Sigma_{2}$. Let us assume that $P$ belongs to the intersection of $\Sigma_{1}$ and $\Sigma_{2}$. If $\alpha_{1}(\tau)$ is a curve in $\Sigma_{1}$ with $\alpha_{1}(0)=P$ and $\alpha_{2}(\tau)=\Phi\left(\alpha_{1}(\tau)\right)$, then the vectors $\alpha_{1}^{\prime}(0), \alpha_{2}^{\prime}(0)$ and $V(P)$ are linearly dependent.

Lemma C.2. At any point in $\mathcal{C}$, the tangent vectors to the Hugoniot, the Hugoniot' ${ }^{\prime}$ and the rarefaction curves are linearly dependent. At a point on the inflection locus, the tangent vector to the composite curve also lies in the same plane as the tangent vectors to the rarefaction curve and to the Hugoniot' curve.

Proof. For points in $\mathcal{C}$, we substitute $X=0$ in equations (A.3) and A.4 and solve for the tangents to the Hugoniot and Hugoniot' curves, respectively. The tangent to the rarefaction curve is given by equations (A.6). In system (A.3), adding the third equation to $-Z$ times the second, we get $Z d \bar{U}-d \bar{V}=0$. The same conclusion is valid for system (A.4), hence the linear dependence of the three vectors. For composites, we apply Lemma C.1 taking $V$ as the tangent vector to the Hugoniot ${ }^{\prime}$ curves, $\Sigma=\mathcal{C}, \Sigma_{1}=S_{o n}^{\prime}, \alpha$ as the rarefaction curve, $\Phi$ as $\psi^{\prime}$ and $\alpha_{1}$ as the composite curve (remember that the composites are the pullbacks of rarefactions by $\psi$ ).

The first part of Lemma C.2 (on Hugoniot curves) was brought to our attention many years ago by C. Gutierrez, as mentioned in [10].

Proof of Theorem 5.1. We divide the proof into two parts:

Piecewise differentiability. To prove that each surface $\mathcal{W}_{\mathcal{S}_{1}}^{\prime}, \mathcal{W}_{\mathcal{R}_{1}}^{\prime}$ and $\mathcal{W}_{\mathcal{C} O_{1}}^{\prime}$ is differentiable we need regularity conditions $I, I I$ and $I I I$, which are stated when needed in the proof; of course, conditions $I, I I$ and $I I I$ are valid for quadratic flux functions.

We assume that the gradient of $G$ is nowhere zero, so that $G=0$ is a well defined manifold; this is certainly the case for quadratic flux functions and their perturbations in the $C^{3}$-Whitney topology.

First we consider $\mathcal{W}_{\mathcal{S}_{1}}^{\prime}$, the saturation by Hugoniot' curves of a 1 -shock curve. We begin with a point $\left(\bar{U}_{0}, \bar{V}_{0}, X_{0}, Z_{0}\right)$ in the 1-shock curve specified by $\left(u_{s}, v_{s}\right)$ and consider $(\bar{U}, \bar{V}, X, Z)$ in the Hugoniot' ${ }^{\prime}$ curve through $\left(\bar{U}_{0}, \bar{V}_{0}, X_{0}, Z_{0}\right)$. The surface $\mathcal{W}_{\mathcal{S}_{1}}^{\prime}$ will 
be described by a system of 6 equations in the 8 variables $\left(\bar{U}_{0}, \bar{V}_{0}, X_{0}, Z_{0}, \bar{U}, \bar{V}, X, Z\right)$. The first 3 equations (1-shock curves) are obtained from system A.5 upon replacing $(\bar{U}, \bar{V}, X, Z)$ by $\left(\bar{U}_{0}, \bar{V}_{0}, X_{0}, Z_{0}\right)$. The three remaining equations (Hugoniot' curves) are also obtained from system (A.5) upon replacing $X$ by $-X, u_{s}$ by $\bar{U}_{0}-X_{0} / 2$ and $v_{s}$ by $\bar{V}_{0}-Z_{0} X_{0} / 2$ in the last two equations.

Solving the four equations that do not involve $G$ for $\bar{U}_{0}, \bar{V}_{0}, X_{0}$ and $Z_{0}$, we obtain $\left(\bar{U}_{0}, \bar{V}_{0}, X_{0}, Z_{0}\right)=\left(p_{1}, p_{2}, p_{3}, p_{4}\right)(\bar{U}, \bar{V}, X, Z)$. Thus we end up with 2 equations in $\bar{U}, \bar{V}, X$ and $Z$. One of the equations is just $G(\bar{U}, \bar{V}, X, Z)=0$ and the other is $G\left(p_{1}, p_{2}, p_{3}, p_{4}\right)=0$. We assume that zero is a regular value for the map from $\mathbb{R}^{3} \times S^{1}$ to $\mathbb{R}^{2}$ defined by $\left(G, G\left(p_{1}, p_{2}, p_{3}, p_{4}\right)\right)(\bar{U}, \bar{V}, X, Z)$; this is the regularity condition $I$.

For the surface $\mathcal{W}_{\mathcal{R}_{1}}^{\prime}$, which is the saturation by Hugoniot' curves of a 1-rarefaction, the proof is similar. We replace system (A.5) by parametric equations for rarefaction curves, $\bar{U}_{0}=R_{1}(\eta), \bar{V}_{0}=R_{2}(\eta), X_{0}=0$ and $Z_{0}=R_{3}(\eta)$. Upon replacing $X$ by $-X$, $u_{s}$ by $R_{1}(\eta)$, and $v_{s}$ by $R_{2}(\eta)$ in the last two equations of system (A.5), we get a system of 3 equations in the 5 variables $(\bar{U}, \bar{V}, X, Z, \eta)$. We assume that the 3 equations have linearly independent gradients; this is the regularity condition $I I$.

The proof for the surface $\mathcal{W}_{\mathcal{C} O_{1}}^{\prime}$, the saturation by Hugoniot' curves of a 1-composite, uses the same procedure as for $\mathcal{W}_{\mathcal{R}_{1}}^{\prime}$, replacing the functions $R_{i}(\eta)$ by $\mathcal{C} O_{i}(\eta), i=1,2,3$. Again we will have 3 equations in the 5 variables $\bar{U}, \bar{V}, X, Z$ and $\eta$, which need to have linearly independent gradients; this is the regularity condition $I I I$.

The three conditions above are valid for $C^{3}$-Whitney perturbations of quadratic flux functions.

Differentiability at transitions. For the sake of brevity we make use of the language of differential forms [1. The list that follows was established in Section 4.2

(a) Transition from $\mathcal{W}_{\mathcal{R}_{1}}^{\prime}$ to $\mathcal{W}_{\mathcal{S}_{1}}^{\prime}$. Let $\mathcal{U}$ be a point in $\mathcal{C}$. Let $\mathcal{W}_{\mathcal{S}_{1}}^{\prime}$ be the intermediate surface built from the 1 -shock curve through $\mathcal{U}$, and $\mathcal{W}_{\mathcal{R}_{1}}^{\prime}$ the intermediate surface built from the rarefaction curve defined by $\mathcal{U}$. The intersection of $\mathcal{W}_{\mathcal{R}_{1}}^{\prime}$ and $\mathcal{W}_{\mathcal{S}_{1}}^{\prime}$ occurs along the Hugoniot' curve through $\mathcal{U}$. From Lemma C.2 at $\mathcal{U}$ the tangents

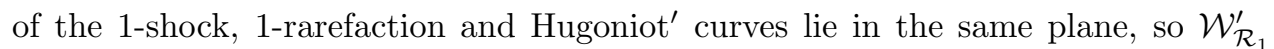
and $\mathcal{W}_{\mathcal{S}_{1}}^{\prime}$ are tangent at this point. Let $\Psi_{\tau}$ be the diffeomorphism that shifts the parameter of Hugoniot' curves by $\tau$. Then $\mathcal{W}_{\mathcal{S}_{1}}^{\prime}$ and $\mathcal{W}_{\mathcal{R}_{1}}^{\prime}$ can be generated from the 1-shock and 1-rarefaction curves of $\mathcal{U}$ by applying $\Psi_{\tau}$ for all $\tau$. The coincidence of the tangent planes of $\mathcal{W}_{\mathcal{R}_{1}}^{\prime}$ and $\mathcal{W}_{\mathcal{S}_{1}}^{\prime}$ at $\mathcal{U}$ is preserved by the diffeomorphism $\Psi_{\tau}$.

(b) Transition from $\mathcal{W}_{\mathcal{R}_{1}}^{\prime}$ to $\mathcal{W}_{\mathcal{C} O_{1}}^{\prime}$. We replace $\mathcal{W}_{\mathcal{S}_{1}}^{\prime}$ by $\mathcal{W}_{\mathcal{C} O_{1}}^{\prime}$ and apply the same argument as above. The difference between (a) and (b) is that in the latter the transition point $\mathcal{U}$ lies in $S o n^{\prime}$.

(c) Transition from $\mathcal{W}_{\mathcal{C O}_{1}}^{\prime}$ to $\mathcal{W}_{\mathcal{S}_{1}}^{\prime}$. Here we begin by proving that the tangent vectors of the 1-shock, Hugoniot ${ }^{\prime}$ and 1-composite curves at $\mathcal{U}$ are linearly dependent. Let $S h, S h^{\prime}$ and $C o$ be these vectors. Let $\omega=Z d \bar{U}-d \bar{V}$ be the differential form defining the rarefaction line field in $\mathcal{C}$ (see (A.6)). Denoting by $R$ the tangent vector to the rarefaction curve, we have $\omega(R)=0$. The vector $S h$ is obtained by solving system (A.3) and the vector $S h^{\prime}$ is obtained by solving system (A.4). The vector $C o$ is obtained by solving system (A.7) (here we make use of this system by considering the natural extension of $\psi$ to a neighborhood of $S o n^{\prime}$ in $\mathcal{M}^{3}$ ). We want to show 
that there exist real numbers $a$ and $b$ such that $C o=a S h+b S h^{\prime}$, i.e., such that the vector $a S h+b S h^{\prime}$ is a solution of system (A.7). The first equation of system (A.7) is trivially satisfied by $S h$ and $S h^{\prime}$. So we have a homogeneous linear system in the unknowns $a$ and $b$ :

$$
\left\{\begin{array}{c}
a \psi^{*}(\omega(S h))+b \psi^{*}\left(\omega\left(S h^{\prime}\right)\right)=0 \\
a d S^{\prime}(S h)+b d S^{\prime}\left(S h^{\prime}\right)=0,
\end{array}\right.
$$

for which we want a non-trivial solution. The determinant of system (C.1 must vanish, and since $d S^{\prime}\left(S h^{\prime}\right)=0$ because of the definition of $S h^{\prime}$, the determinant is just the product $-\psi^{*}\left(\omega\left(S h^{\prime}\right)\right) d S^{\prime}(S h)$. Let us show that $\psi^{*}\left(\omega\left(S h^{\prime}\right)\right)=0$. On $\mathcal{C}$, the vectors $R, S h$ and $S h^{\prime}$ are linearly dependent, so $S h^{\prime}=c S h+e R$ for some unknown $c$ and $e$. As $R$ is in the kernel of $\omega, \omega\left(S h^{\prime}\right)=c \omega(S h)+e \omega(R)=c \omega(S h)$. Taking the pullback, we get $\psi^{*}\left(\omega\left(S h^{\prime}\right)\right)=c \psi^{*}(\omega(S h))$, and it suffices to show that $\psi^{*}(\omega(S h))=0$. Since $\mathcal{C}$ is defined by $X=0$, the second and third equations in (A.3) become $d \bar{U}=0$ and $d \bar{V}=0$. So, $(Z d \bar{U}-d \bar{V})(S h)=0$, which implies $\psi^{*}((Z d \bar{U}-d \bar{V})(S h))=0$, so that the vectors $C o, S h, S h^{\prime}$ are linearly dependent in $S o n^{\prime}$. To complete the proof that the transition from $\mathcal{W}_{\mathcal{S}_{1}}^{\prime}$ to $\mathcal{W}_{\mathcal{C} O_{1}}^{\prime}$ is smooth we use the same argument as in case (a).

(d) Transition from $\mathcal{W}_{\mathcal{S}_{1}}^{\prime}$ to $\mathcal{W}_{\mathcal{R}_{1}}^{\prime}$. In this case a 1-shock curve arriving at Son is followed by a Hugoniot' curve connecting $\operatorname{Son}$ to $\mathcal{C}$ and then followed by a rarefaction, i.e., at this transition $\mathcal{W}_{\mathcal{S}_{1}}^{\prime}$ and $\mathcal{W}_{\mathcal{R}_{1}}^{\prime}$ intersect along the Hugoniot' ${ }^{\prime}$ curve connecting Son to $\mathcal{C}$. We use the natural extension of the map $\psi^{\prime}$ (defined in Subsection 4.1 when discussing composite curves) to a neighborhood of Son in $\mathcal{M}^{3}$ to pull back the rarefaction curves into a family of curves in Son, which we may call composite', since they are the analogues of the composite curves defined in Son'. The argument used in (c) to prove that the tangent vectors to the 1-shock, Hugoniot' and 1-composite curves are linearly dependent at points in $S o n^{\prime}$ applies to prove that the tangent vectors to the 1-shock, Hugoniot' and composite' curves are linearly dependent at points in Son. The rest of the proof is the same as in case (c), using $\psi^{\prime}$ instead of $\psi$.

(e) Consider a (non-local) 1-shock curve $\mathcal{S}_{1}\left(\mathcal{Q}_{1}\right)$ through $\mathcal{Q}_{1}$. From cases (c) and $(\mathrm{d})$, we know that the transitions from $\mathcal{W}_{\mathcal{S}_{1}}^{\prime}\left(\mathcal{Q}_{1}\right)$ to $\mathcal{W}_{\mathcal{C} O_{1}}^{\prime}\left(\mathcal{U}_{L}\right)$ and from $\mathcal{W}_{\mathcal{S}_{1}}^{\prime}\left(\mathcal{Q}_{1}\right)$ to $\mathcal{W}_{\mathcal{R}_{1}}^{\prime}\left(\mathcal{Q}_{2}\right)$ are differentiable along $s h^{\prime}\left(\mathcal{Q}_{1}\right)$. Therefore the transition from $\mathcal{W}_{\mathcal{C} O_{1}}^{\prime}\left(\mathcal{U}_{L}\right)$ to $\mathcal{W}_{\mathcal{R}_{1}}^{\prime}\left(\mathcal{Q}_{2}\right)$ is also differentiabe along $\operatorname{sh}^{\prime}\left(\mathcal{Q}_{1}\right)$.

REMARK C.3. The projection of the smooth intersection $\mathcal{W}_{\mathcal{S}_{1}}^{\prime} \cap \mathcal{W}_{\mathcal{C} O_{1}}^{\prime}$ onto state space along Hugoniot' curves is a point that satisfies the hypothesis of the Bethe-Wendroff theorem.

D. The Bethe-Wendroff theorem. In this appendix we state the version of the Bethe-Wendroff theorem in Furtado's doctoral thesis [5]. Furtado constructed oneparameter families of composite waves by combining the differential equations for a rarefaction curve with the Rankine-Hugoniot conditions, following [9]. 
Consider the states $U_{0}(\xi)$ in the rarefaction portion of the composite wave for $\xi$ between $\xi_{0}$ and $\eta$, where $U_{0}(\xi)$ satisfies $U_{\xi}=r_{i}(U)$ and $r_{i}(U)$ is the right eigenvector of $d F$. Let $U$ denote the state on the opposite side of the discontinuity from $U_{0}(\eta)$. Then the Rankine-Hugoniot conditions are satisfied:

$$
-s(\eta)\left[U-U_{0}(\eta)\right]+F(U)-F\left(U_{0}(\eta)\right)=0, \text { with } s(\eta)=\lambda_{i}\left(U_{0}(\eta)\right),
$$

where $\lambda_{i}\left(U_{0}(\eta)\right)$ denotes the eigenvalue and $s(\eta)$ the shock speed. The composite locus is specified by $U$ and $\eta$ satisfying (D.1). Therefore we define the composite locus based on the given rarefaction curve to consist of states $U$ such that (D.1) holds for some $\eta$. The composite locus may be obtained by projecting the zero-set of the function that appears on the left hand side of (D.1) onto state space. Since the differential of the function in (D.1),

$$
-\left[U-U_{0}(\eta)\right] s^{\prime}(\eta) d \eta+[-s(\eta)+d F(U)] d U,
$$

is nonzero, the implicit function theorem guarantees that the composite locus is a onedimensional manifold in a neighborhood of $(U, \eta)$ unless, for some $j$,

$$
\begin{aligned}
s(\eta)= & \lambda_{j}(U) \quad \text { and } \\
& \text { either } l_{j}(U) .\left(U-U_{0}(\eta)\right)=0 \quad \text { or } s^{\prime}(\eta)=\nabla \lambda_{j}\left(U_{0}(\eta)\right) \dot{r}_{j}\left(U_{0}(\eta)\right)=0 .
\end{aligned}
$$

Moreover, (D.2) implies that the $U$ component of the tangent vector $d U$ to the zero-set must be nonzero if $U \neq U_{0}(\eta)$ and $s^{\prime}(\eta) \neq 0$. In this case, the state space projection of the zero-set near such a point is a smooth one-dimensional manifold.

Away from bifurcation points, both the Rankine-Hugoniot and the composite loci may be parametrized by a single variable. The following theorem describes the qualitative behavior of $s(U)$.

Theorem D.1. Consider either $(i)$ the Hugoniot locus through a state $U_{0}$ or $(i i)$ a composite locus based on a rarefaction of the $i$ th family. Let $U$ be a point on the locus and assume that

$$
s^{*}=\lambda_{i}\left(U^{*}\right) \quad \text { and } \quad l_{i}\left(U^{*}\right) \cdot\left(U^{*}-U_{0}\right)=0
$$

do not hold at $U$ if the locus is the Hugoniot locus, or that (D.3) and (D.4) do not hold at $U$ if the locus is a composite locus. Then the following are equivalent:

(a) $s^{\prime}(U)=0$;

(b) $\lambda_{j}(U)=s(U)$ for some $j$.

In this case, $\lambda_{j}(U)-s(U)$ and $\dot{s}(U)$ vanish to the same order and the locus is tangent to a rarefaction curve of the $j$ th family.

This theorem was first proved in [19] for the Hugoniot loci and then extended in [13. to composite loci.

Acknowledgments. We thank J. Glimm for constant encouragement in the course of this research program. Dan Marchesin is grateful to Frederico Furtado, with whom he had many discussions about the definition of wave curves and Riemann solutions in the wave manifold. Furtado's doctoral thesis [5] contains many results on wave curves in state space that were essential at the early stages of this research. We also thank V. Matos for his careful proofreading of the manuscript, and for suggestions on how to 
improve the text. The authors gratefully acknowledge the hospitality of IMPA, PUC-Rio, UFMG and UnB during the development of this work.

\section{REFERENCES}

[1] V. Arnol'd, Mathematical methods of classical mechanics, Graduate Texts in Math., vol. 60, Springer-Verlag, New York-Heidelberg-Berlin, 1978. MR.0690288 (57:14033b)

[2] J. Bastos-Gonçalves and H. Reis, The geometry of quadratic $2 \times 2$ systems of conservation laws, Acta Applicandae Mathematicae 88 (2005), 269-329. MR2192058 (2007f:35182)

[3] C. S. Eschenazi, Rarefaction fields in systems of two and three conservation laws, Doctoral thesis, Dep. de Matemática, PUC-Rio, 1992 (in Portuguese).

[4] C. S. Eschenazi and C. F. B. Palmeira, The structure of composite rarefaction-shock foliations for quadratic systems of conservation laws, Matemática Contemporânea 22 (2002), 113-140. MR 1965790 (2004e:35145)

[5] F. Furtado, Structural stability of nonlinear waves for conservation laws, Ph.D. thesis, New York Univ., 1989.

[6] I. M. Gel'fand, Some problems in the theory of quasilinear equations, Amer. Math. Soc. Transl., ser. 229 (1963), 295-381, English translation. MR0153960 (27:3921)

[7] C. Gutierrez and J. Sotomayor, Structural stability of configurations of lines of principal curvature, Asterisque 98-99 (1982), 195-215. MR724448 (85h:58006)

[8] E. Isaacson, D. Marchesin, C. F. Palmeira, and B. Plohr, A global formalism for nonlinear waves in conservation laws, Comm. Math. Phys. 146 (1992), 505-552. MR.1167301 (93h:35124)

[9] T.-P. Liu, The Riemann problem for general $2 \times 2$ conservation laws, Trans. Amer. Math. Soc. 199 (1974), 89-112. MR0367472(51:3714)

[10] D. Marchesin and C. F. B. Palmeira, Topology of elementary waves for mixed-type systems of conservation laws, Journal of Dynamics and Differential Equations 6 (1994), no. 3, 421-440. MR.1298810 (95i:35181)

[11] D. Marchesin, B. Plohr, and S. Schecter, Structurally stable Riemann solutions, Journal of Differential Equations 126 (1996), 303-354. MR1383980(97d:35136)

[12] Classifications of codimension-one Riemann solutions, Journal of Dynamics and Differential Equations 13 (2001), no. 3, 523-588. MR1845094(2002f:35162)

[13] R. Menikoff and B. Plohr, The Riemann problem for fluid flow of real materials, Rev. Mod. Phys. 61 (1989), 75-130. MR977944 (90a:35142)

[14] O. Oleinik, On the uniqueness of the generalized solution of a Cauchy problem for a nonlinear system of equations occurring in mechanics, Uspekhi Mat. Nauk 73 (1957), 169-176. MR0094543 (20:1057)

[15] C. F. Palmeira, Line fields defined by eigenspaces of derivatives of maps from the plane to itself, Proceedings of the VIth International Colloquium on Differential Geometry (1988), 177-205, Universidad de Santiago de Compostela, 1989. MR1040846 (91a:58099)

[16] D. Schaeffer and M. Shearer, The classification of $2 \times 2$ systems of non-strictly hyperbolic conservation laws, with application to oil recovery (with appendix by D. Marchesin, P.J. Paes Leme, D.G. Schaeffer, and M. Shearer), Comm. Pure Appl. Math. 40 (1987), 141-178. MR872382 (88a:35155)

[17] Z. Tang and T. Ting, Wave curves for Riemann problems of plane waves in simple isotropic elastic solids, Internat. J. Engrg. Sci. 25 (1987), 1343-1381. MR921358 (88m:73011)

[18] Solution of a Riemann problem for elasticity, Journal of Elasticity 26 (1991), 43-63.

[19] B. Wendroff, The Riemann problem for materials with non-convex equations of state: I, isentropic flow; II, general flow, J. Math. Anal. Appl. 38 (1972), 454-466; 640-658. 